\title{
A low complexity selected mapping scheme for peak to average power ratio reduction with digital predistortion in OFDM systems
}

\begin{abstract}
One of the effective methods used for reducing peak-to-average power ratio (PAPR) in orthogonal frequency division multiplexing (OFDM) systems is selected mapping (SLM). In this paper, a new SLM scheme called DSI-SLM, which is a combination of dummy sequence insertion (DSI) and conventional selected mapping (C-SLM) is proposed. Previous techniques have had some drawbacks. In DSI, increasing the number of dummy sequences to have better PAPR degrades transmission efficiency, and in C-SLM, the complexity rises dramatically when the number of sub-blocks increases. The proposed DSI-SLM scheme significantly reduces the complexity because of the reduction in the number of sub-blocks compared with the C-SLM technique while its PAPR performance is even better. To enhance the efficiency of the OFDM system and suppress the out-of-band distortion from the power amplifier nonlinearity, a digital predistortion technique is applied to the DSI-SLM scheme. Simulations are carried out with the actual power amplifier model and the OFDM signal based on the worldwide interoperability for microwave access standard and quadrature phaseshift keying modulation. The simulation results show improvement in PAPR reduction and complexity, whereas the BER performance is slightly worse.
\end{abstract}

Keyword: Digital predistortion; Dummy sequence insertion; OFDM; PAPR; Selected map 